\title{
Keyword Index Volume 32 (2013)
}

accent-based

acoustic analysis

acoustic neuroma

acquired brain damage

acquired brain injury

activities of daily living

adaption

ADHD

adults

AFO

aged

aggression

aging

ambulation

ankle clonus

ankle foot orthosis

aphasia

assessment

assistive device

asymmetry

attention

attention control

autonomic dysfunction

balance confidence

balance disorders

balance

battery

benign paroxysmal positional vertigo

Berg balance scale

bi-factor model

biopsy

blast forces

blood oxygen saturation

Bonferroni

Botulinum toxin

boxing

brain injury literature

brain injury rehabilitation

brain plasticity

brain-derived neurotrophic factor (BDNF)

brainstem

carer stress

carrying weight
185 centralization 649

649 cerebellum 493

455 cerebral infarct 583

771 cerebral palsy $1,9,17,287$

103 challenges and opportunities $\quad 751$

233 challenging behaviour 761

311 child problems $\quad 59$

555 children 751

751 choir 929

317 CHRIST 287

965 chronic stroke 323, 591

761 chronic traumatic encephalopathy 211

225, 233, 409 circadian rhythm 337

635 closed-kinetic chain exercise 385

635 cognition disorders 729

377 cognitive assessment 253

$\begin{array}{lll}157,573,899,915,929 & \text { cognitive performance } & 687\end{array}$

699, 875 cognitive rehabilitation $\quad 297$

$\begin{array}{ll}317,833 \text { cognitive } & 359\end{array}$

563 communication disorders 351

33 communication $\quad 929$

555 community based rehabilitation 781

327 community-based ambulation training 425

compensation $\quad 311$

117 computer 359

445 concussed athlete and treatment 543

$323,473,493,957$ concussion 211, 463, 473, 519, 543

157 consciousness 823

455 coping 721

9 cortical activation 279

253 cortical disinhibition 833

601 corticoreticular pathway 305, 655

397 corticoreticulospinal tract 305, 655, 861

687 corticospinal tract 27, 305, 583, 655, 861

693 cost-effectiveness 781

635 Critical-1llness-Polyneuropathy 149

$\begin{array}{ll}117,211 \text { cross-sectional area } & 17\end{array}$

95 cupula 437

27 deglutition disorders 949

573 dementia 199

493 DEMMI scale 957

developmental stuttering 297

351 diffusion tensor imaging 27, 305, 583, 665

563 disability $\quad 69,699$ 
Disability and Health

disequilibrium

dizziness

dorsal stream and ventral stream

dorsolateral prefrontal cortex

Downs and Black

dysexecutive syndrome

early neurological rehabilitation

education

EEG

effort

electrical stimulation of the urinary bladder

electromyography

emotional problems

endurance

epidemiology

ethics

exercise

exertion

existential approach

falls prevention

family health

fatigue

feedback monitoring

five-times-sit-to-stand test

fMRI

foot wedge

football

frontotemporolimbic damage

functional independence measure

functional NIRS

\section{gait}

gait ataxia

gait pattern

gait training

generalization

Glasgow coma scale

Glasgow outcome scale

gross motor function

gym

hand paresis, ischemic stroke

heart rate

heart rate recovery

hematoma

hemiparesis

hemiparetic $\mathrm{CP}$
729 hemisphere 609

483 highly concentrated oxygen 687

445, 455, 473, 529 holistic approaches 751

483 holistic rehabilitation 791

823 Holm's sequential 693

95 hospitalization 233

823 hypertonicity 885

149 ICF 729

671 illness-related variables $\quad 59$

279 imbalance 455

415 inner ear 455

867 intellectually and developmentally disabled people 687

149, 609 interdisciplinary assessment and interventions 351

771 inter-limb transfer 609

655 International Classification of Functioning 729

875 inter-patient variability 721

275 ipisilateral motor cortex 617

$117,125,141,327$ ischemic stroke 391,573

415 isokinetic 601

771 kinematic analysis $\quad 591$

473 kinocilium 437

59

$\begin{array}{ll}655,875 \text { labyrinthitis } & 455 \\ 823 & 915\end{array}$

823 language 915

9 language trajectory 165

279 Latin America 679

563 line bisection $\quad 839$

211 long-term outcomes 225

707

671 macula 437

861 magnetoencephalagraphy 617

maladaption 311

17, 377, 635 manual dexterity 943

445 measurement 729

385 mechanisms 721

135, 317 Meniere's disease 455

899 mental health 679

409 meta-analysis 359

409 middle cerebral artery $\quad 583$

17 migraine 455

125 mild traumatic brain injury 397, 473

Mirror illusion $\quad 617$

573 mixed types of dysarthria 185

687 mobility measurement 957

327 mobility outcome 957

87 modern psychometrics 253

369 mood 929

563 mortality 225, 233 
motor control

motor function

motor imagery training

motor recovery

motor suppression

movement training

multiple sclerosis

muscle stiffness

muscle strength

music

music therapy

music-speech protocol development

myopathy

861

outcomes

671

$1,369,583$

$377,415,655,943$

287, 415

National Alzheimer's Coordinating Center (NACC) 199

neglect

neglect rehabilitation

nerve conduction studies

neurobehavioral functioning

neurobehavioural

neurobehavioural disorder

neurocognitive assessment

NEurocognitive Joyful Attentive

Training Intervention (NEJATI)

neurogenic urinary bladder dysfunction

neuroimaging

neurological surgery

neuro-optometric rehabilitation therapy

neuropsychological deficits

neuropsychological examinations

neuropsychological rehabilitation

neuropsychological training

neuropsychology

neurorehabilitation

nitric oxide

non-fluent aphasia

nystagmus

33,839

699, 791

751,839

253,693

455,493

older adults

online

open loop pointing

open-kinetic chain exercises

operant learning theory

orthosis

orthostatic hypotension

orthotic devices

osteopathy

ototoxicity

outcome

outcomes measures
43

311

191

625

833

33

929

185

601

43

149

729

781

473

297

867

707

817

483

555

275

555

791

141

915

oxidized LDL receptor gene 1

parent's functioning $\quad 59$

parental ABI $\quad 59$

paresis $\quad 87$

paretic limb

Parkinson disease

563

Parkinson's disease 125, 135, 141, 949

patient education

817

patients

635

peak torque $\quad 601$

pediatric vestibular deficits $\quad 507$

pediatric vestibular rehabilitation $\quad 507$

pediatric vestibular testing $\quad 507$

perception 415

$\begin{array}{ll}\text { physical fitness } & 327\end{array}$

physiotherapy 135

plantar pressure distribution $\quad 385$

plasticity 311

positron emission tomography imaging 397

post-concussion $\quad 397$

posterior fossa tumor $\quad 165$

$\begin{array}{ll}\text { prediction } & 803\end{array}$

primary motor cortex $\quad 191$

prism adaptation $\quad 839$

prognosis 87, 345, 391

prospective memory 103

$\begin{array}{ll}\text { psychosocial functioning } & 267\end{array}$

psychosocial issues $\quad 351$

$\begin{array}{ll}\text { psychotherapy } & 771\end{array}$

quality assessment tools 95

quality of life $\quad 117,125,337$

questionnaires $\quad 729$

rating scale 415

267 reaching movement 591

817 readability 817

839 recovery 87

385 rehabilitation $27,33,69,103,233,377,573$,

$761 \quad 591,625,635,655,721,761$,

$317 \quad 817,915,949,967$

529 reliability 157

377 repetitive transcranial magnetic stimulation (rTMS) 573

135 review 359, 781

529 right-hemispheric brain lesion 43

803 risk-adapted treatments 165

463 robotic-assisted gait training 833
PEDro $\quad 95$ 
robotics

rTMS

running

saccule

segmental muscle vibration

semantic feature treatment

semicircular ducts

sensory deficits

sensory retraining

severity

sexual function

sexuality

sexual therapy

singing

skeletal muscle

sleep-wake

social brain

social support

spasticity

spatial neglect

spinal cord injury

spinal injuries

splinting

sports injury

stereocilia

stop-signal task

straight ahead

stretch

stretching

striola

stroke patients

stroke

$27,33,43,87,305,311,327,337,345$,
$1,359,369,377,625,655,833,915,929$

superior canal dehiscence syndrome

surface electromyography

susceptibility

systematic

TBI caregivers

TBI

technology

telephone

tests

theory of mind

Thera-band

therapies

therapy

943

943

233

69

929

601

337

707

125

$1,369,885$

425
625 timed up and go

823 TMS

915

17 topographical map $\quad 279$

transcranial direct current stimulation $\quad 191$

437 transcranial magnetic stimulation 915

591 Traumatic brain injuries 233

899 traumatic brain injury (TBI) 199

437 traumatic brain injury $69,211,225,267,409,463$,

treatment $519,671,699,707,721,729$,

$751,771,781,791,803,875$

69 type I error 693

43

upper extremity

$287,345,415$

upper limb

885

urodynamic examination $\quad 867$

utricle

437

validity 157

ventilator therapy 149

vertigo

$445,455,493,529$

vestibular

437

885 vestibular and balance dysfunction $\quad 543$

473 vestibular apparatus 437

437 vestibular dysfunction 445

191 vestibular neuritis 455

839 vestibular rehabilitation therapy 543

369 vestibular rehabilitation 463, 519

625 vestibular nuclear complex 437

437 vestibular ocular reflex 437, 455, 483

185 vestibular spinal reflex 437

vibration platform $\quad 655$

vibration therapy 1

455 virtual reality 103

415 vision 493

391 visual-vestibular disorder 483

359 visuospatial training 43

VOR 483

679 vowel space area 649

445
$781 \quad$ walking ability $\quad 655$

253 walking 425, 967

729 WBV 655

707 weight bearing 563

317 well-being 929

233 whole body vibration 655

359 word retrieval 899 